\title{
Políticas públicas urbanas para la Reducción del Riesgo de Desastres en contextos de marginalidad. El caso del Bajo Luján, Mendoza, Argentina
}

\section{Urban public policies for Disaster Risk Reduction in marginal contexts. The case of Bajo Luján, Mendoza, Argentina}

\begin{abstract}
Silvia Graciela Quiroga ${ }^{1}{ }^{1}$ Universidad Nacional de Cuyo. Facultad de Filosofía y Letras. Municipalidad de Luján de Cuyo. Centro de Estrategias Territoriales para el Mercosur. Mendoza, Argentina. *Autor de correspondencia: silgquiroga@gmail.com.
\end{abstract}

Recibido en versión modificada: 10 setiembre 2021

Quiroga, S. G. (2021). Políticas públicas urbanas para la Reducción del Riesgo de Desastres en contextos de marginalidad. El caso del Bajo Luján, Mendoza, Argentina. Investigaciones y Estudios - UNA, 12

(2), 48-58. https://doi.org/10.47133/IEUNA2125b

Resumen. La marginalidad urbana, entendida no sólo como un patrón de asentamiento en el territorio, sino como una condición social y económica caracterizada por la pobreza, la precariedad y la exclusión social; conlleva a la existencia de condiciones de riesgo que se manifiestan no sólo en las áreas marginales, sino que influyen en vastos contextos urbanos en Argentina y América Latina. El artículo explora en primer lugar, los factores de riesgo existentes en la urbanización marginal llamada "Bajo de Luján”. En segundo lugar se analizan los fundamentos y propuestas de cambio radical de esta zona, en el contexto del Plan Ribera Luján, impulsados por el Municipio de Luján de Cuyo y el Gobierno de la Provincia de Mendoza (2017). Por último, se reflexiona sobre los aspectos científicos, normativos, institucionales, financieros $\mathrm{y}$ comunitarios que han permitido reconvertir -a través de las políticas públicas- las condiciones de riesgo preexistentes en una oportunidad de renovación urbana con influencia metropolitana en el Gran Mendoza.

Palabras clave. marginación, riesgo, oportunidad, políticas públicas.

Abstract. Urban marginality, understood not only as a pattern of settlement in the territory, but as a social and economic condition characterized by poverty, precariousness and social exclusion; It leads to the existence of risk conditions that manifest themselves not only in marginal areas, but also influence vast urban contexts in Argentina and Latin America. The article explores in the first place, the risk factors existing in the marginal urbanization called "Bajo de Luján". Secondly, the foundations and proposals for radical change in this area are analyzed, in the context of the Ribera Luján Plan, promoted by the Municipality of Luján de Cuyo and the Government of the Province of 
Mendoza. Finally, it reflects on the scientific, regulatory, institutional, financial and community aspects that have allowed to reconvert -through public policies- pre-existing risk conditions in an urban renewal opportunity with metropolitan influence in Greater Mendoza.

Keywords. marginalization, riesgo, opportunity, public policies.

\section{INTRODUCCIÓN}

La búsqueda de nuevos mecanismos para la Reducción del Riesgo de Desastres (RRD) en contextos de marginalidad urbana conlleva a la consideración de múltiples factores que se relacionan con la vida de las ciudades. Aspectos como la ocupación ilegal de tierras, la degradación del ambiente, la localización de población en sitios expuestos a amenazas, la ineficiencia en la provisión de servicios, la precariedad en las redes de conectividad, la pobreza y la marginación social son algunas de las consideraciones que aparecen con mayor frecuencia en la bibliografía sobre el tema.

Sin embargo, un aspecto poco explorado en estos espacios marginales es la constitución de escenarios de riesgo que derivan de estas condiciones de vida y que pueden proyectarse hacia el resto de la ciudad.

En este contexto dinámico, los gobiernos locales impulsan políticas públicas como el Ordenamiento Territorial y la Gestión Ambiental que difícilmente pueden revertir los procesos de marginalidad; especialmente cuando los daños ambientales y los riesgos para la sociedad ya están materializados en el territorio.
Frente a esta problemática, algunas experiencias latinoamericanas enfocadas a la gestión territorial sostenible indican que para revertir los escenarios de riesgo es necesario tomar medidas más profundas, complejas y mitigadoras; tales como la relocalización de poblaciones, la recuperación de áreas con daño ambiental grave, la inversión en infraestructuras $y$ equipamientos vitales $y$ especialmente la construcción de un nuevo acuerdo social que posibilite una mejor localización de estas poblaciones vulnerables en la vida de la ciudad.

En el presente artículo se analiza el caso del Barrio del Bajo Luján, el cual se emplazaba en el lecho del Río Mendoza; espacio que fue transformado radicalmente por el Plan Ribera Luján. Esta iniciativa incluyó la relocalización de la población, la remediación ambiental y con ello, la reducción notable de los riesgos urbanos que afectaban los sectores marginales de la Ciudad de Luján de Cuyo.

Como resultado de la reflexión se busca identificar cuáles son los factores de orden científico, institucional, normativo, organizacional, financiero y comunitario que en el corto plazo posibilitarán la reducción del riesgo y la renovación urbana de una ciudad mediana de importancia estratégica en la Provincia de Mendoza y en el Corredor Bioceánico Central del Mercosur.

Planteamiento del problema. Las ciudades latinoamericanas experimentan rápidos procesos de crecimiento que muchas veces no son previstos por los mecanismos de Ordenamiento del Territorio, ni están al alcance del poder de control de los municipios; en especial para frenar la transformación de las periferias urbanas en lugares no aptos para la urbanización (terrenos fiscales, zonas inundables, áreas contaminadas, entre otras). 
En Argentina existen lineamientos para el Ordenamiento Territorial a nivel nacional. Sin embargo, las autonomías provinciales reconocen que corresponde a los gobiernos locales la responsabilidad de formular los planes de OT a nivel local; a fin de prever los espacios de crecimiento urbano, la creación de las áreas industriales, el resguardo de las zonas productivas y de valor estratégico por sus recursos naturales y culturales.

Frente a ello, la localización y crecimiento de población en zonas marginales - en condiciones de informalidad y pobreza- genera escenarios de riesgo urbano cuyos efectos trascienden a estas periferias urbanas y se proyectan en vastos territorios. En el caso del Bajo Luján, las consecuencias derivadas de la contaminación de suelos y del agua superficial y subterránea pueden manifestarse aguas debajo de la Cuenca del Río Mendoza.

Por lo general, cuando aparecen estos asentamientos en zonas marginales, los municipios no cuentan con herramientas eficientes que les permitan responder en tiempo real a estos rápidos procesos; siendo necesarias medidas extraordinarias de intervención territorial para revertir las condiciones de riesgo, daño ambiental y económico; tales como la coordinación de múltiples actores institucionales, provisión de fondos y un profundo trabajo con las comunidades afectadas, que en sus lugares de origen no cuentan con servicios y oportunidades de trabajo, educación y salud, entre otros.

Sin lugar a duda, las renovaciones urbanas, tanto como las relocalizaciones de población deben considerar un diagnóstico previo exhaustivo que permita identificar los escenarios de riesgo, las limitaciones y las potencialidades de los nuevos terrenos a urbanizar.

Objetivo. La investigación tiene como objetivo identificar qué factores de orden científico, técnico, legal, institucional, financiero y comunitario son eficientes para revertir una condición de riesgo local en una oportunidad, mediante procesos de recuperación y renovación urbana; tanto como de relocalización de poblaciones vulnerables y expuestas a múltiples amenazas.

Hipótesis. La implementación de políticas públicas enfocadas a la renovación urbana sustentable -en el marco de la filosofía de las ciudades inteligentes- constituye una oportunidad para reducir las condiciones de riesgo de poblaciones marginales que cuentan con la posibilidad de ser relocalizadas en terrenos adaptados a los nuevos requerimientos urbanos.

Área de estudio. Se toma como área de estudio el sector sur de la ciudad del Luján de Cuyo, ubicada en el sector centro- oeste de la República Argentina. Es la capital del Departamento homónimo y forma parte de la aglomeración del Gran Mendoza. Esta metrópoli es la ciudad principal del oeste argentino y por su posición brinda servicios estratégicos al Corredor Bioceánico Central del Mercosur (Ruta Nacional 7), que vincula los puertos del Atlántico y del Pacífico, a través de la Cordillera de los Andes Centrales argentino-chilenos.

En las últimas décadas la Ciudad de Luján de Cuyo ha experimentado un importante crecimiento poblacional, contando en el año 2010 con 27594 habitantes, según el Censo Nacional de Población, Hogares y Vivienda de ese año. El crecimiento poblacional se ha hecho evidente por el surgimiento de numerosos barrios y loteos, tanto como por el 
crecimiento urbanizaciones marginales; siendo la más crítica el denominado Barrio Bajo Luján. Esta urbanización surgió hace siete décadas en el lecho del Rio Mendoza (área propensa a inundaciones) y ha experimentado un proceso de crecimiento físico y poblacional que no ha sido posible de encauzar ni frenar por parte del municipio. Como resultado, en el Bajo Luján llegaron a vivir 750 familias, que se agrupaban en 5 barrios, cubriendo en total una superficie de aproximadamente 65 has. Las características del asentamiento eran heterogéneas y muy precarias, donde era posible observar viviendas construidas con materiales reciclados (chapas, madera, adobe, ladrillos) con diferentes técnicas de construcción y terminación. (Figura 1).

En este proceso de crecimiento urbano marginal, las sucesivas gestiones municipales encararon el problema del Bajo Lujan con enfoques muy diferentes, que han permitido reconocer cortes temporales:

Período 1960-2010: en estos años la ciudad miró con perplejidad y descontento el crecimiento del Bajo Luján, sin tomar demasiadas cartas en el asunto. Las intervenciones del sector público se concretaban cuando se producían delitos, incidentes de inseguridad urbana en su periferia o asistencia durante inundaciones periódicas que requerían la evacuación frecuente de los pobladores del barrio.

Período 2011-2015: el gobierno municipal de turno -bajo las presiones mediáticas, sociales y políticas consideró seriamente la idea de consolidar la urbanización del Bajo Luján adentro del lecho del río, con inversión pública destinada a la mejora de viviendas, caminos, construcción de infraestructura urbana y equipamientos comunitarios.

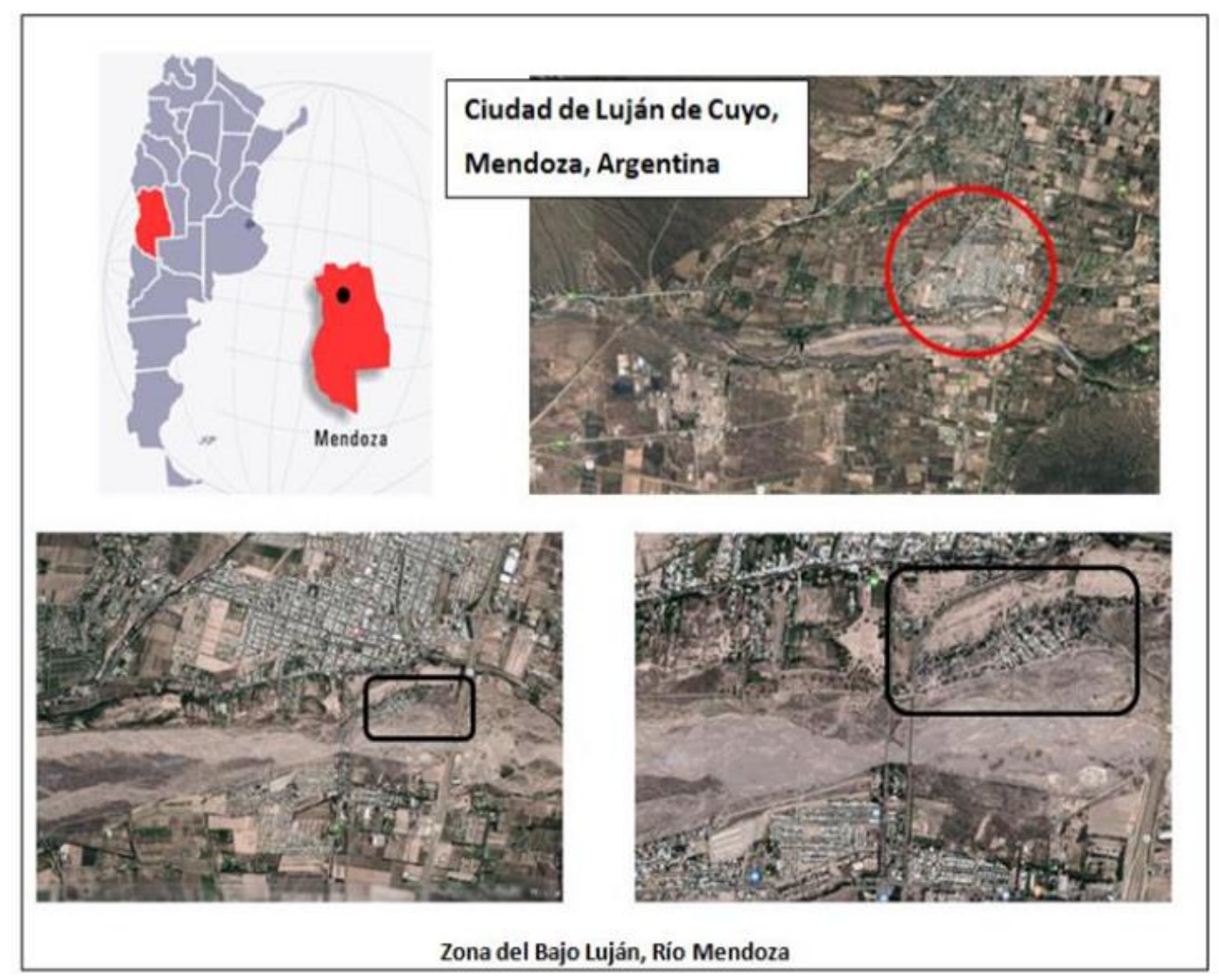

Figura 1. Zona del Bajo Luján. Mendoza, Argentina. 
Estas propuestas apostaron por una mejora de las condiciones de vida "in situ" y no prosperaron a la hora de conseguir validación técnica de otras instituciones gubernamentales y sectoriales, respecto a las condiciones de riesgo del sitio, con lo cual se imposibilitó el financiamiento necesario.

Período 2016 en adelante: el municipio elaboró su Plan de Ordenamiento Territorial con enfoque de Reducción de Riesgo de Desastres (RRD) en el marco de la Ley 8051/2009 e impulsó un conjunto amplio de medidas de intervención territorial en la Ciudad de Luján. Entre ellas, evaluó la condición de riesgo del Bajo Luján, considerándola inaceptable.

Ello dio pie, junto con otro conjunto de propuestas, al surgimiento de una iniciativa integral de renovación urbana denominada Plan Rivera Luján, que contempla criterios de sustentabilidad ambiental, resguardo de patrimonio, implementación de procesos participativos y mejora sustancial en las condiciones de seguridad, funcionamiento y estética urbana.

Metodología utilizada. Para el estudio de los escenarios de riesgo en la Ciudad de Luján de Cuyo y su área de influencia, se consideran metodologías enfocadas a detectar condiciones de riesgo subyacente. Se toman como antecedentes los trabajos realizados en la última década por Quiroga y otros (2016), tanto como los aportes provenientes de diversos organismos técnicos y académicos de la Provincia de Mendoza.

Se utilizaron diversas fuentes de información bibliográfica, documental y cartográfica. Se realizaron trabajos de relevamiento del área de estudio y se diseñaron instrumentos de consulta (entrevistas) para el análisis de la vulnerabilidad y la percepción del riesgo por parte de la población lujanina.
En cuanto al tema de las políticas públicas, se realizaron encuentros con funcionarios de gobierno y representantes de instituciones participantes en el proceso de relocalización del Bajo Luján.

Entre los antecedentes más relevantes para el estudio de amenazas, se consideraron los resultados de la modelización de erogación de caudales de la Presa Potrerillos elaborada por la Empresa CEMPPSA, para diferentes escenarios de operación y rotura de presa. En la Figura 2 se puede observar el área de inundación esperada en el caso de descargas de caudales desde la Presa Potrerillos.

Asimismo, en el recuadro puede observarse la localización del Bajo Luján en el área de impacto de la inundación esperada.

Con la finalidad de construir escenarios hipotéticos de riesgo urbano, y en especial del Bajo Luján, se realizó un análisis de amenazas sísmicas, hidrológicas, contaminantes y tecnológicas, cuyas áreas de impacto fueron contrastadas con un estudio de la vulnerabilidad de la población, que contempló la identificación de instalaciones vitales expuestas y la percepción social del riesgo por parte de los lujaninos. Se tomó como antecedente principal el trabajo sobre aspectos objetivos y subjetivos del riesgo en la ciudad de Luján de Cuyo elaborado por Quiroga et al. (2017). Como resultado pudo caracterizarse el riesgo en diferentes sectores territoriales de la Ciudad de Luján de Cuyo, en los cuales fue posible identificar actores, instituciones y posibles medidas de intervención para realizar una RRD.

La evaluación de las condiciones diferenciales de riesgo en el territorio, tanto como la población expuesta y su capacidad de recuperación, puso en evidencia que la situación del Bajo Luján era inaceptable y se requería de intervención 


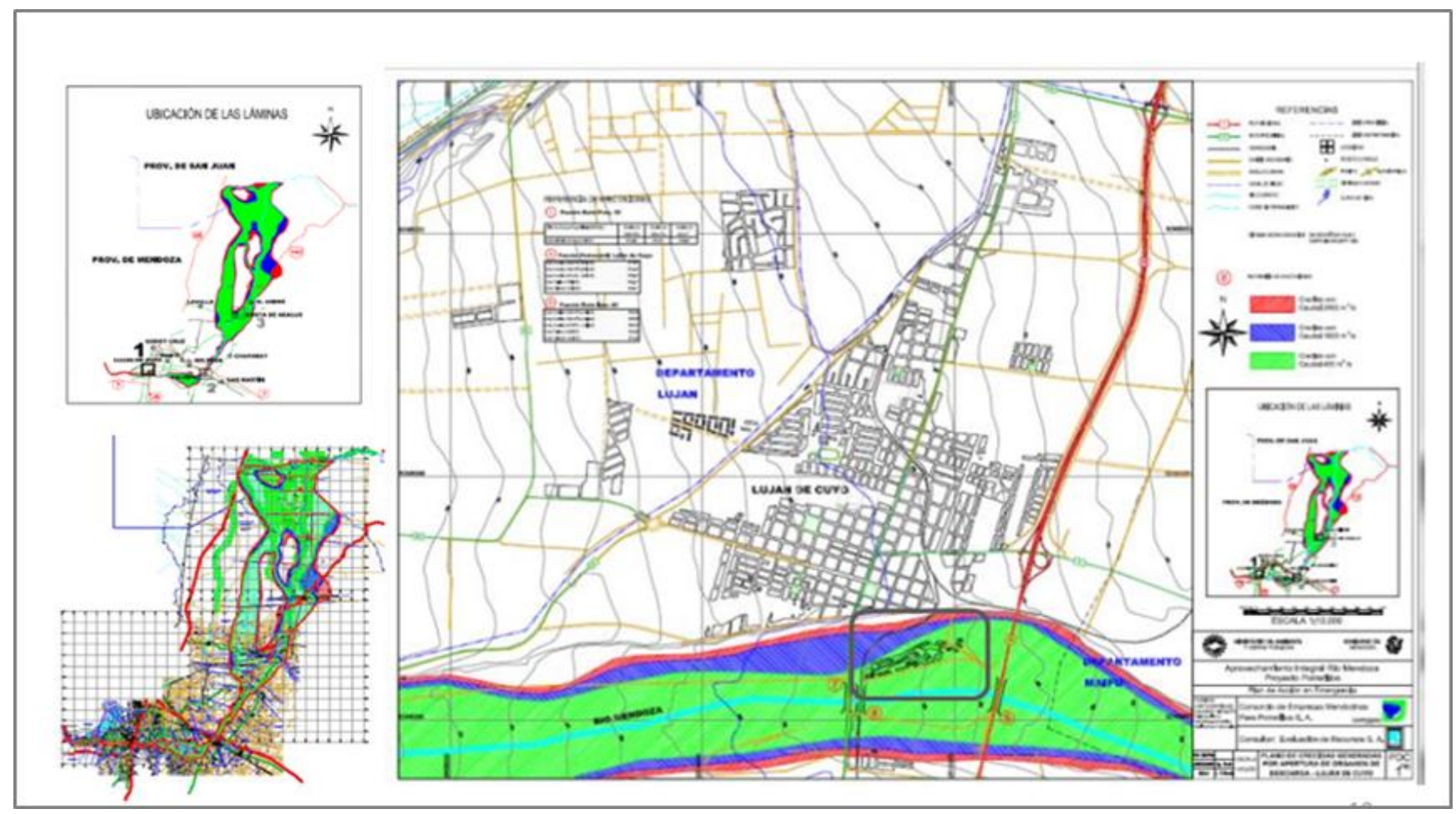

Figura 2. Área de impacto de crecidas del Río Mendoza, provocadas por operación de órganos de descarga de la Presa Potrerillos. Fuente: CEMPPSA (2013).

planificada por parte del estado, en sus diferentes niveles, especialmente el nivel local (Figura 3).

\section{Plan Ribera Luján: renovación urbana y} RRD a través de políticas públicas locales. De esta forma, una vez detectada la necesidad y la urgencia de intervenir, se puso en marcha el Plan Ribera Luján. Esta iniciativa permitió realizar un trabajo con la comunidad del Bajo Luján por parte del Municipio y de otras instituciones de gobierno de la Provincia de Mendoza (2017).

Se realizaron relevamientos de terreno, consultas a los vecinos, talleres de concientización y censos de población de los asentamientos a relocalizar. A través de múltiples estrategias participativas y de sensibilización, las familias expresaron su consenso respecto al proyecto de relocalización de sus viviendas, que implicó el surgimiento de 12 nuevos barrios en otros distritos de Luján de Cuyo.
El proceso relocalización de asentamientos, planificación urbana y remediación ambiental fue liderado por el Municipio Luján de Cuyo, que en forma distintiva implementó políticas públicas territoriales que se manifestaron en una nueva zonificación de usos del suelo para cicatrizar y poner en valor toda la ribera del Río Mendoza a la altura de la Ciudad de Luján y en la relocalización propiamente dicha de las poblaciones de los asentamientos en riesgo (Figura 4, 5 y 6).

Este ejemplo de políticas públicas territoriales implementadas a nivel local pone en evidencia la importancia del acceso al financiamiento, tanto como la capacidad operativa y de diálogo del Municipio con otras instituciones locales, provinciales y nacionales que tienen jurisdicción en su territorio. 


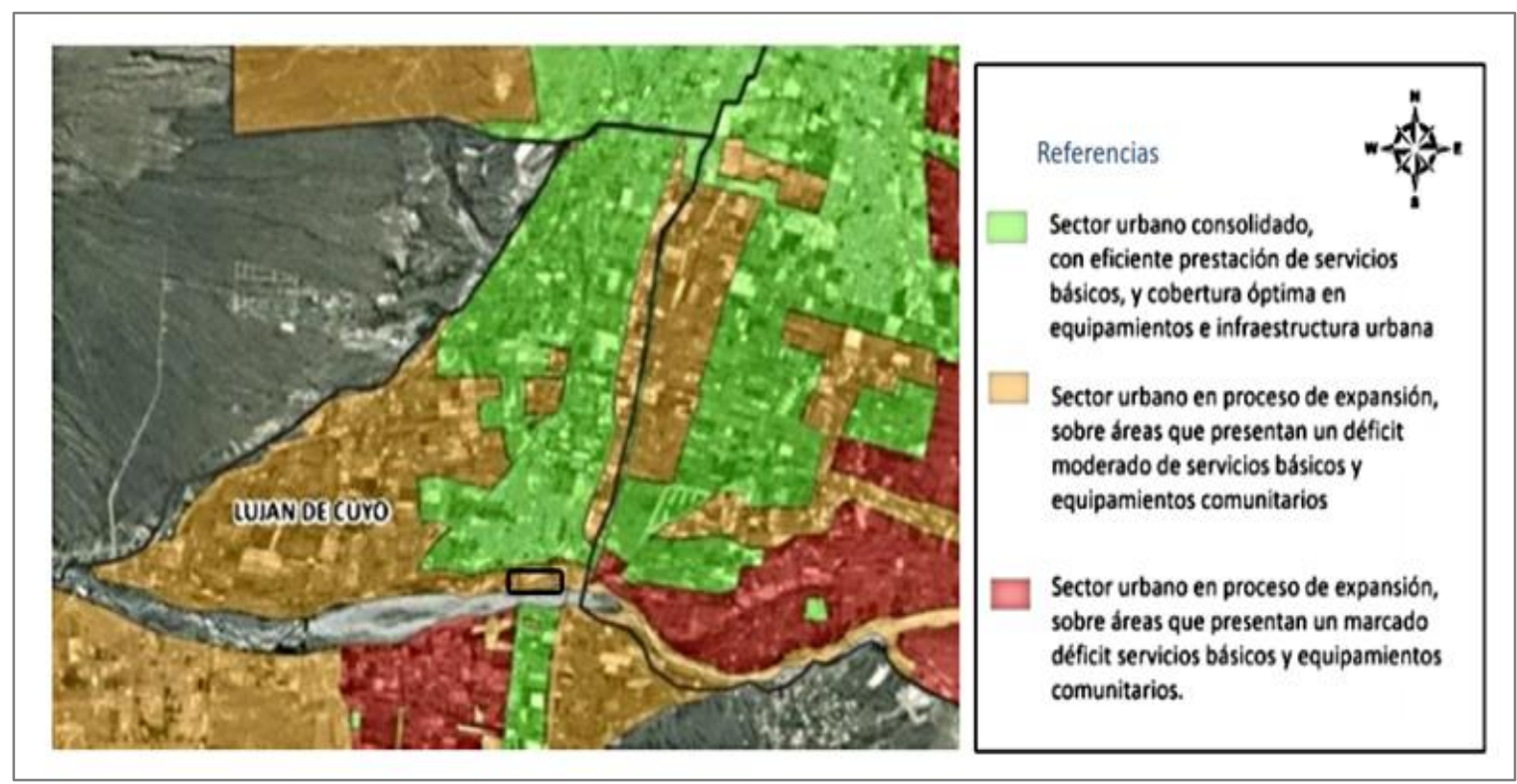

Figura 3. Condiciones de vulnerabilidad de la población asociados a expansión urbana, dotación de servicios y equipamientos. Fuente: Quiroga y Bustamante (2017).

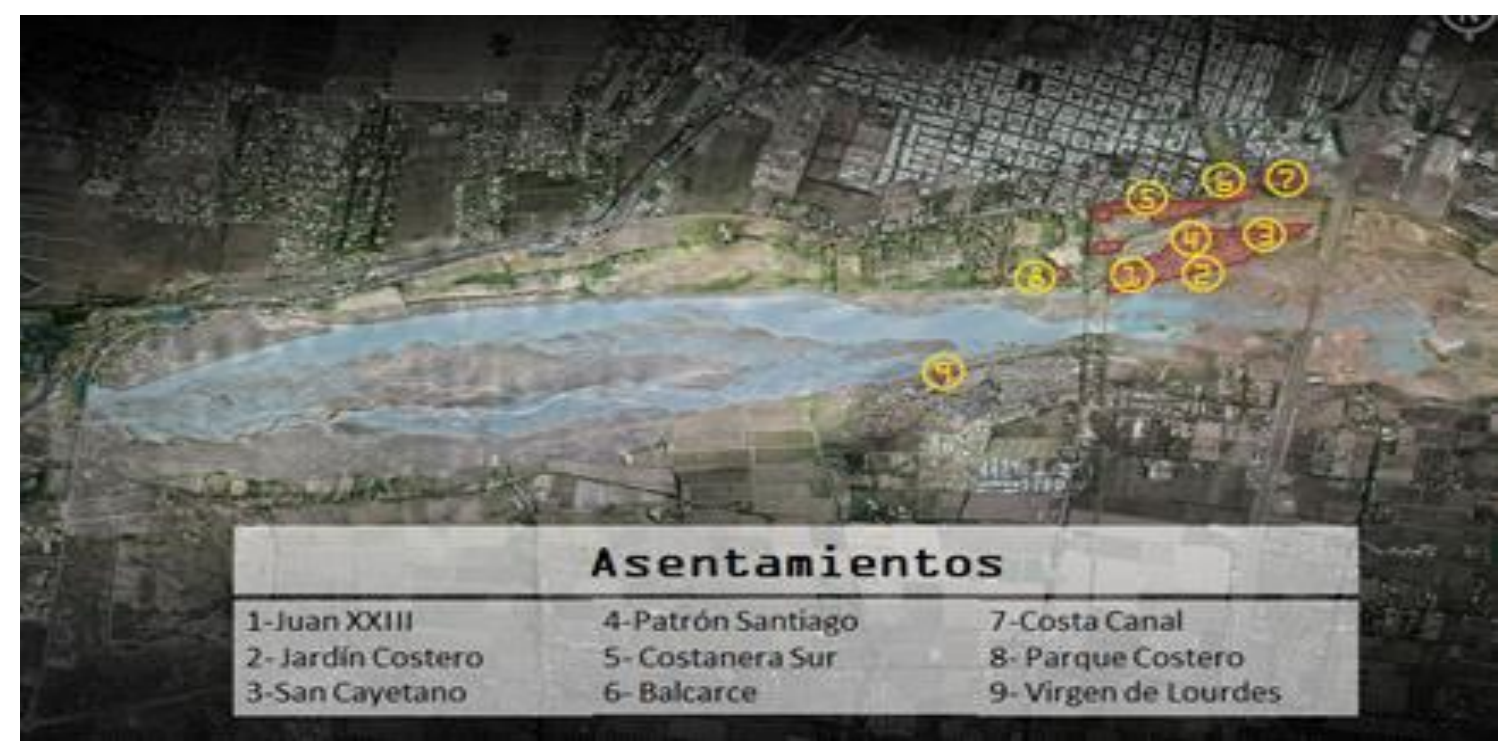

Figura 4. Asentamientos marginales del Bajo Luján. Fuente: Gobierno de Mendoza. Municipalidad de Luján de Cuyo (2017). Proyecto Ribera Luján.

El resultado permitió no sólo realizar una renovación urbana, sino principalmente desactivar un potente escenario de riesgo urbano latente durante décadas en el sector sur de la Ciudad de Luján de Cuyo.

Una mención especial merece el trabajo detallado, respetuoso y participativo que se realizó con las comunidades que fueron relocalizadas en nuevos barrios ubicados en los distritos de Perdriel y Agrelo.

Otro aspecto de gran importancia que derivó del Plan Ribera Luján es que, debido a sus múltiples facetas y complejidad, produjo cambios significativos en la institución municipal, que se vio enriquecida por el trabajo coordinado e interdisciplinario, logrando nuevas capacidades en 
la planificación, gestión y administración de áreas urbanas del departamento.

Estas capacidades, materializados en el territorio, permitieron cambiar el rumbo de los acontecimientos, antes de que se produjera un desastre con las consiguientes pérdidas inaceptables de vidas y bienes.

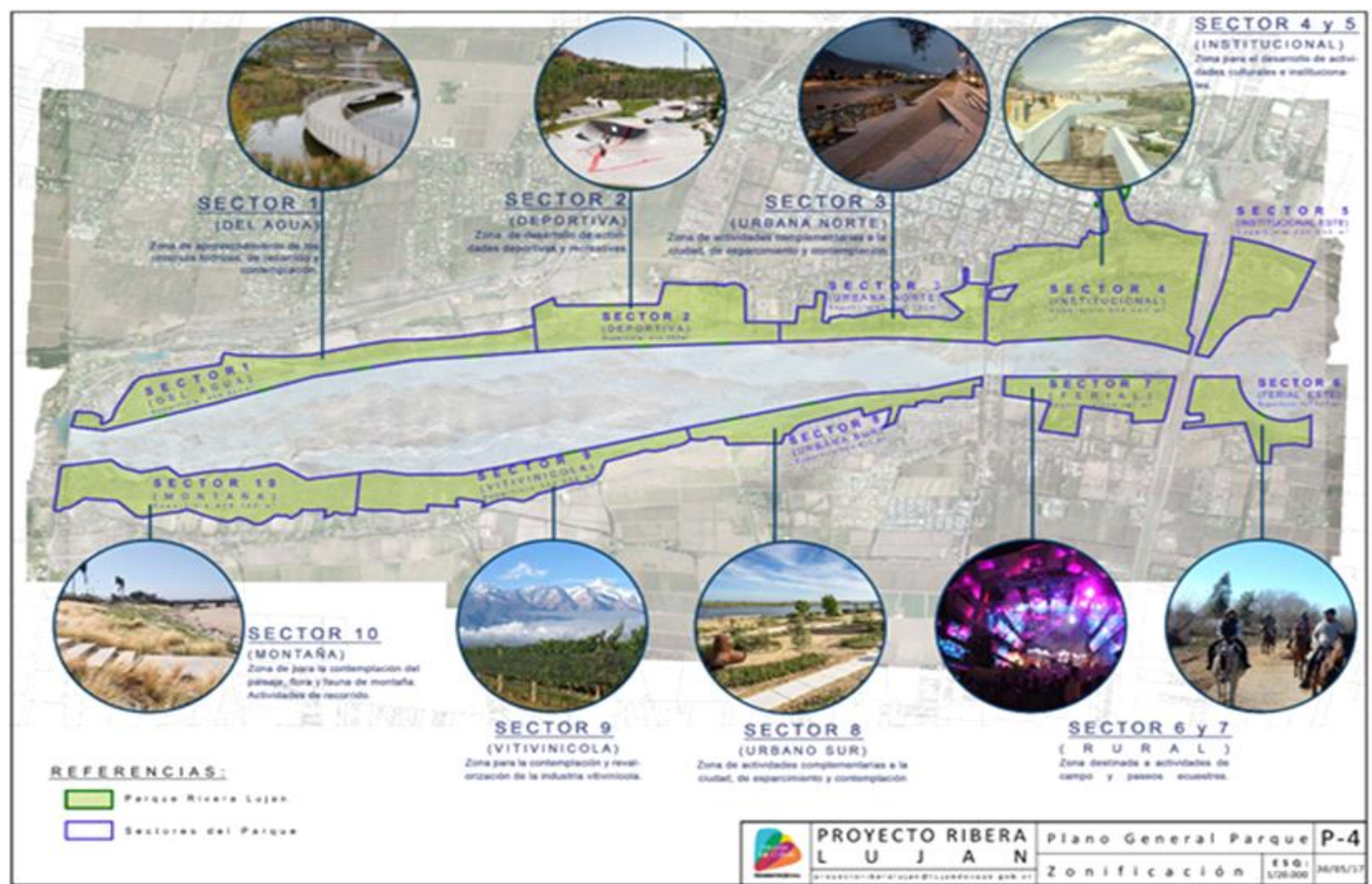

Figura 5. Zonificación propuesta. Fuente: Gobierno de Mendoza. Municipalidad de Luján de Cuyo (2017). Proyecto Ribera Luján.

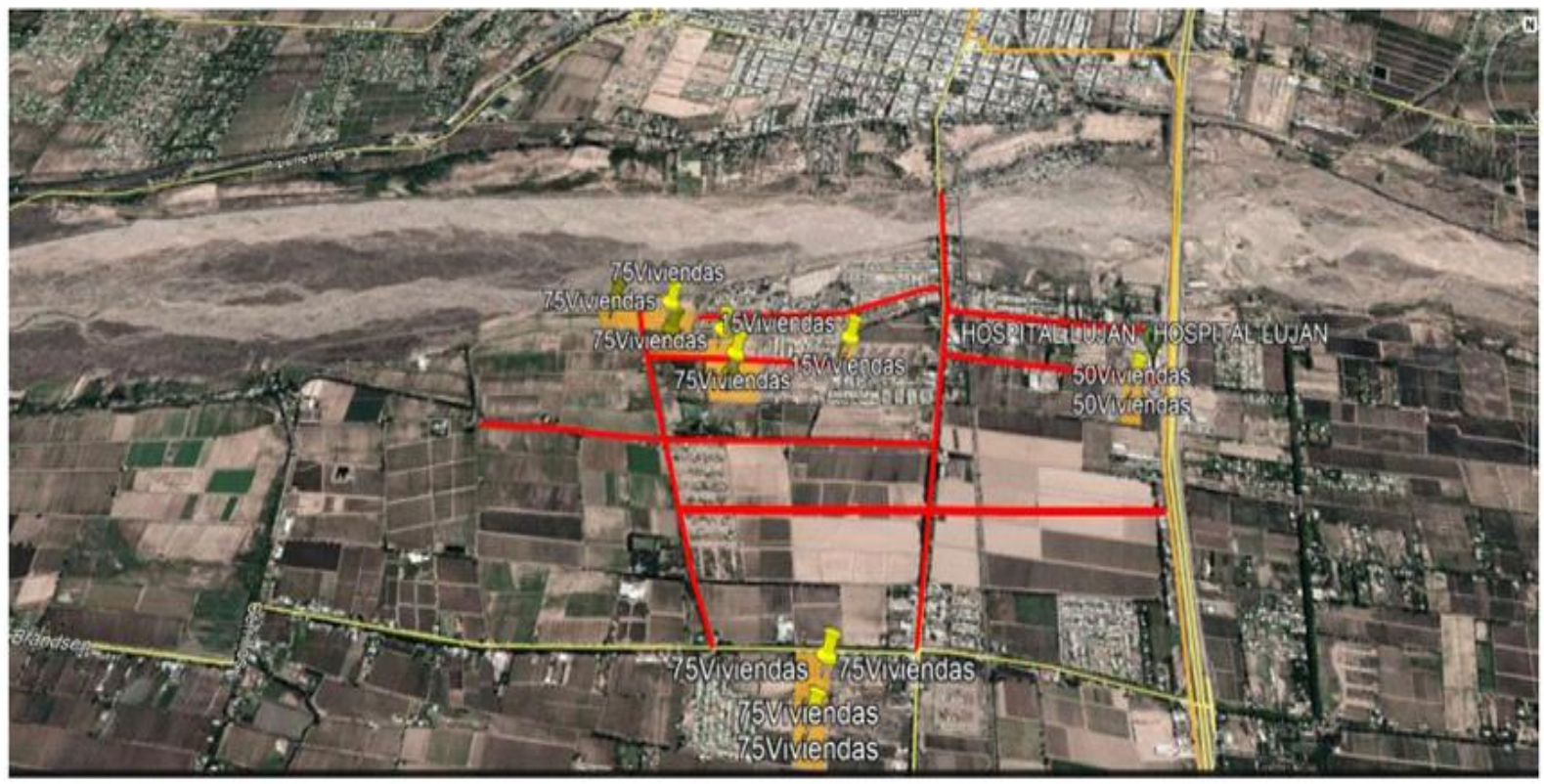

Figura 6. Relocalización de asentamientos y nueva trama vial propuesta en el Distrito Perdriel. Fuente: Gobierno de Mendoza. Municipalidad de Luján de Cuyo (2017). Proyecto Ribera Luján. 
Resultados obtenidos. La metodología aplicada ha permitido confirmar, en primer lugar, que las condiciones de riesgo del Bajo Luján eran muy altas, debido a la precaria situación de sus habitantes, con relación a las variables consideradas: población, vivienda, empleo, servicios (educación, salud, seguridad), recreación y cultura. Según un estudio antecedente, el Bajo Luján quedaba definido como un sector urbano en proceso de expansión sobre áreas que presentan un déficit moderado de servicios básicos y equipamiento (Quiroga et al., 2016).

Respecto a los escenarios de riesgo posibles de materializarse en el área de estudio, se constató la exposición del Bajo Luján a múltiples amenazas de origen natural y tecnológico. Los eventos que se manifestaban con una frecuencia anual eran las inundaciones causadas por precipitaciones y crecidas del Río Mendoza, los sismos, los procesos de contaminación ambiental, la aparición de enfermedades respiratorias y digestivas crónicas, la inseguridad y delincuencia urbana.

En un segundo nivel de recurrencia, aparecían las inundaciones causadas por maniobras en las compuertas de la Presa Potrerillos, las enfermedades epidémicas transmitidas por vectores, los deslizamientos de suelos y los incendios en viviendas y depósitos informales de basura.

En tercer lugar, los escenarios posibles pero remotos mostraban la ocurrencia de grandes inundaciones destructivas por la rotura de la Presa Potrerillos y explosiones contaminantes por contingencias en el Distrito Industrial Luján de Cuyo.

Asimismo, las condiciones de vida del asentamiento marginal evidenciaban unos elevados niveles de vulnerabilidad, caracterizada por la pobreza, la precariedad laboral, el desempleo, la informalidad en la tenencia de bienes, la desnutrición, el analfabetismo, la falta de cohesión interna y con el resto de la Ciudad de Luján de Cuyo, que producía un efecto fuerte de segregación urbana.

En cuanto al conocimiento del riesgo, las encuestas realizadas a pobladores de la Ciudad de Luján y del Bajo Luján en especial, demostraron un desfasaje entre las amenazas que la población manifestaba conocer y las amenazas a las que estaba realmente expuesta. Asimismo, los patrones de organización y comportamiento no reflejaban una conciencia clara del riesgo existente. En especial, en el Barrio Bajo Luján, la comunidad se encontraba inmersa en la preocupación por la supervivencia diaria, lo que restaba importancia a otros escenarios de riesgo, vistos como lejanos o no posibles de materializarse.

Asimismo, las políticas públicas implementadas a través del Plan Ribera Luján muestran la importancia de realizar una mirada amplia sobre el territorio, que permite identificar escenarios de riesgo latentes $\mathrm{y}$ con ello, plantear nuevas preguntas, acciones y desafíos hacia el futuro.

Para concluir, puede comprobarse que el proceso de relocalización poblacional, renovación urbana y RRD ha sido posible por la incidencia de múltiples factores:

Científico-técnicos. existencia de estudios técnicos sectoriales, diagnósticos sobre escenarios de riesgo, modelizaciones y publicaciones que permitieron profundizar y tomar conciencia de esa situación en la Ciudad de Luján de Cuyo.

Normativos. la Provincia de Mendoza cuenta con la Ley 8051 de Ordenamiento Territorial y Uso del Suelo, que incorpora a los planes de gestión del 
riesgo como instrumentos del OT, en concordancia con los principios del Marco de Sendai. Esta ley establece que los municipios deben elaborar sus propios Planes Municipales de Ordenamiento Territorial, tomando las premisas del Plan Provincial.

Institucionales. el Municipio de Luján de Cuyo ha desarrollado una labor de diagnóstico, planificación y gestión territorial de varias décadas, en el cual se incorporaron diversos actores de otros ámbitos, realizando alianzas estratégicas con instituciones locales, provinciales, nacionales e internacionales.

Técnicos. las capacidades profesionales del municipio fueron complementadas con aportes de especialistas del ámbito académico, científico y gubernamental. Ello posibilitó plantear soluciones más integrales y complejas, con el apoyo de las instituciones implicadas.

Financieros. el ejercicio de la planificación estratégica y la profundización del Ordenamiento Territorial con enfoque de RRD permitió el desarrollo de un plan urbano innovador que contribuyó al desarrollo sostenible y a la seguridad de la población. Como es sabido, estas premisas son esenciales en las evaluaciones de proyectos que realizan los organismos de financiamiento, sean nacionales o internacionales. En el caso del Plan Ribera Luján se contó con financiamiento local, nacional e internacional proveniente del BID.

Comunitarios. la experiencia de RRD a nivel local demuestra que el trabajo con las comunidades es un elemento esencial para que las acciones sean eficientes. Crear canales de comunicación innovadores, fortalecer la organización comunitaria, identificar los posibles escenarios de riesgo, capacitar a las familias, las mujeres y los niños para la autoprotección frente a diferentes adversidades, propiciar la inserción laboral de los jóvenes y mejorar la calidad de vida de las familias de los asentamientos precarios, han sido los desafíos más profundos de este proyecto.

En síntesis, la metodología utilizada y el caso de estudio seleccionado permitieron cumplir con el objetivo propuesto y a la vez, verificar la hipótesis planteada.

El caso analizado permite visualizar que un escenario de riesgo urbano marginal no puede ser gestionado ni revertido con los instrumentos habituales con que cuentan los gobiernos locales. Por lo contrario, es necesario implementar nuevos enfoques científicos y técnicos para comprender los problemas territoriales, fortaleciendo el trabajo interdisciplinario $y$ en especial, con las comunidades vulnerables y expuestas. Estas bases pueden dar origen a nuevas políticas de incidencia territorial; cuya implementación implica un proceso de RRD, en el que pueden aparecer múltiples actores, nuevos costos aparentes y mayores tiempos para lograr la ejecución de las acciones necesarias.

\section{CONSIDERACIONES FINALES}

Si bien el caso del Bajo Luján muestra una experiencia exitosa en términos de solución de graves problemas de crecimiento informal e inseguridad urbana, lo cierto es que en América Latina estos ejemplos son muy limitados. Predominan los casos en los que el crecimiento urbano marginal avanza sin la posibilidad de ser encauzado por parte de los respectivos gobiernos locales, siendo muy escasos los controles y las alternativas de prestación de servicios básicos. 
En este contexto cabe preguntarse:

¿Qué estrategias podrían utilizar los gobiernos locales para llevar a cabo un reconocimiento precoz de estos procesos en las zonas marginales?

¿Qué acuerdos y herramientas serían necesarios para disponer de una capacidad de reacción adecuada a la velocidad de dichos procesos?

¿En qué forma las universidades, las $\mathrm{ONG}^{\prime} \mathrm{s}$ y otras instituciones pueden colaborar con la detección de escenarios de riesgo urbano?

¿Qué mecanismos de diseño urbano y financiamiento pueden desarrollarse con el fin de intervenir adecuadamente en estos territorios cuyo cambio es rápido y descontrolado?

¿Son suficientes las herramientas de planificación territorial y control que utilizan los municipios o es necesario innovar en este sentido, incorporando con mayor fuerza los diálogos intersectoriales y los procesos participativos?

Estos interrogantes latentes en muchos ámbitos urbanos de América Latina nos desafían a pensar en nuevas formas de relación y participación entre las comunidades, los gobiernos locales, las instituciones y en especial los sectores de ciencia y técnica. Los avances en este diálogo permitirán generar nuevas miradas e instrumentos para prevenir, responder y dar soluciones a los difíciles escenarios de riesgo que se presentan en los ámbitos urbanos marginales.

Declaración de interés. Los autores declaran

\section{REFERENCIAS BIBLIOGRÁFICAS}

CEMPPSA. (2013). Plan de Acción en Emergencia (PAE). PROYECTO Potrerillos. Provincia de Mendoza, Argentina. Consorcio de Empresas Mendocinas para Potrerillos.

Gobierno de Mendoza. (2017). Plan de Ordenamiento Territorial de la Provincia de Mendoza. Caracterización preliminar de las amenazas naturales. Aporte de la Agencia Provincial de Ordenamiento Territorial. Ministerio de Tierras, Ambiente y Recursos Naturales.

Gobierno de Mendoza. Municipalidad de Luján de Cuyo (2017). Proyecto Ribera Luján, Mendoza. Luján de Cuyo, Argentina.

Quiroga, S. G., Bustamante, M. V., Videla, M. A. \& Vila, I. B. (2016). Factores subyacentes del riesgo y Ordenamiento Territorial en el Gran Mendoza, Argentina". Plataforma de información para políticas públicas de la Universidad Nacional de Cuyo. Disponible en: http://www.politicaspublicas.uncu.edu.ar/articulos/index /factores-subyacentes-del-riesgo-y-ordenamientoterritorial-en-el-gran-mendoza-argentina

Quiroga, S. \& Bustamante, M. (2017). La evaluación de riesgos en el Ordenamiento Territorial. En XII Jornadas Cuyanas de Geografía, 19 al 21 de septiembre de 2017. Mendoza, Argentina. Facultad de Filosofía y Letras. Universidad Nacional de Cuyo

no tener conflicto de intereses. 